\title{
CONSULTA DE ENFERMAGEM EM \\ PUERICULTURA À CRIANÇA HAITIANA: \\ DIFICULDADES E POSSIBILIDADES
}

\author{
NURSING CONSULTATION IN CHILDCARE \\ TO HAITIAN CHILDREN: DIFFICULTIES \\ AND POSSIBILITIES
}

\section{CONSULTA DE ENFERMERÍA EN PUERICULTURA AL NIÑO HAITIANO: DIFICULTADES Y POSIBILIDADES}

\author{
Elisangela Argenta Zanatta ${ }^{1}$ \\ Cheila Karei Siega ${ }^{2}$ \\ Ingrid Pujol Hanzen ${ }^{3}$ \\ Luiza Alcântara de Carvalho ${ }^{4}$
}

Como citar este artigo: Zanatta EA, Siega CK, Hanzen IP, Carvalho LA. Consulta de enfermagem em puericultura à criança haitiana: dificuldades e possibilidades. Rev baiana enferm. 2020;34:e35639.

Objetivo: identificar as dificuldades vivenciadas pelos enfermeiros na Consulta de Enfermagem em puericultura à criança haitiana na Atenção Primária à Saúde e as possibilidades para superar esses desafios. Método: estudo exploratório, descritivo com abordagem qualitativa, realizado com dez enfermeiros por meio de entrevista realizada em julho de 2018. Submeteram-se os depoimentos à Análise de Conteúdo. Resultados: a análise dos dados deu origem a duas categorias: Dificuldades na intercomunicação entre enfermeiros e famílias de crianças haitianas e Dificuldades culturais relacionadas ao cuidado da criança haitiana. Conclusão: os resultados revelam dificuldades na realização da Consulta de Enfermagem às crianças, especialmente relacionadas à intercomunicação, pela língua falada pelos haitianos, e pelas questões culturais que permeiam e embasam o cuidado à criança pelas famílias. Para superar as dificuldades os enfermeiros indicam o uso da comunicação não verbal, visita domiciliar para conhecer o contexto e melhorar a confiança e o vínculo com as famílias.

Descritores: Cuidado da Criança. Atenção Primária à Saúde. Processo de Enfermagem. Imigrantes. Consulta de Enfermagem.

Objective: To identify the difficulties lived by the nurses during the Nursing Consultation in childcare to Haitian children in the Primary Health Care and the possibilities to overcome these challenges. Method: this is an exploratory, descriptive study with a qualitative approach, conducted with ten nurses through an interview held in July 2018. The testimonies were submitted to Content Analysis. Results: From the data analysis, two categories emerged: Difficulties in the intercommunication between nurses and families of Haitian children and Cultural difficulties for the care of Haitian children. Conclusion: the results reveal difficulties in carrying out the Nursing Consultation for children, especially related to intercommunication, due to the language spoken by Haitians, and due to the cultural issues

\footnotetext{
Enfermeira e Obstetra. Doutora em Enfermagem. Professora Adjunta da Universidade do Estado de Santa Catarina. Chapecó, Santa Catarina, Brasil. elisangela.zanatta@udesc.br. https://orcid.org/0000-0002-7426-6472.

Enfermeira. Mestre em Enfermagem. Professora do Serviço Nacional de Aprendizagem Comercial. Caçador, Santa Catarina, Brasil. http://orcid.org/0000-000 I$9272-2526$.

3 Enfermeira. Mestre em Enfermagem. Enfermeira da Secretaria Municipal de Saúde de Chapecó. Chapecó, Santa Catarina, Brasil. https://orcid.org/0000-000 I-9808-2005. 4 Estudante de Enfermagem. Universidade do Estado de Santa Catarina. Chapecó, Santa Catarina, Brasil. http://orcid.org/0000-0002-1730-2553.
} 
that permeate and support the care of children by families. To overcome the difficulties, nurses indicate the use of non-verbal communication, home visits to get to know the context and improve trust and bond with the families.

Descriptors: Childcare. Primary Health Care. Nursing Process. Immigrants. Nursing consultation.

Objetivo: identificar las dificultades vividas por los enfermeros en la Consulta de Enfermería en puericultura al niño haitiano en la Atención Primaria a la Salud y las posibilidades para superar esos desafíos. Método: estudio exploratorio, descriptivo con enfoque cualitativo, realizado con diez enfermeros por medio de una entrevista realizada en julio de 2018. Las declaraciones se sometieron al Análisis de Contenido. Resultados: dos categorias surgieron del análisis de los datos: Dificultades en la intercomunicación entre enfermeros y familias de niños haitianos y Dificultades culturales relacionadas al cuidado del niño haitiano. Conclusión: Los resultados muestran dificultades en la realización de la Consulta de Enfermería a los niños, especialmente relacionadas a la intercomunicación, por edioma hablado por los haitianos, y por las cuestiones culturales permeando y basando el cuidado al niño por las familias. Para superar las dificultades, los enfermeros indican el uso de la comunicación no verbal, visita domiciliaria para conocer el contexto y mejorar la confianza y el vínculo con las familias.

Descriptores: Cuidado del Niño. Atención Primaria a la Salud. Proceso de Enfermería. Inmigrantes. Consulta de Enfermería.

\section{Introdução}

A Atenção Primária à Saúde (APS), no Brasil, constitui-se como a principal porta de entrada para o Sistema Único de Saúde (SUS), possuindo papel primordial no cuidado à criança. Compreende cuidados primários capazes de melhorar a qualidade de vida da criança, com foco no crescimento e desenvolvimento adequados sob os aspectos biológicos, psíquicos, afetivos e sociais $^{(1)}$.

O crescimento infantil é caracterizado por um processo biológico, dinâmico e contínuo, constituindo-se um indicador da saúde infantil. De maneira ampla, envolve o aumento da massa corporal e mudanças morfológicas que diferenciam a criança do adulto, influenciadas por diversos fatores como gênero, hereditariedade, meio ambiente, nutrição, dentre outros ${ }^{(2)}$.

O desenvolvimento da criança remete a uma transformação ampla, complexa, dinâmica e contínua, que, além do crescimento, abrange a maturação, a aprendizagem, os aspectos psíquicos e sociais. Considerando esses aspectos, ressalta-se a "importância da família em proporcionar ambiente social e psicológico favorável ao desenvolvimento da criança e à promoção de sua saúde"(2:30).

Nesse contexto, a puericultura tem como objetivos a prevenção de agravos e a promoção da saúde infantil com vistas a garantir o desenvolvimento pleno, sendo operacionalizada por meio da Consulta de Enfermagem (CE) realizada pelo enfermeiro na APS ${ }^{(3)}$.

As atividades realizadas pelos enfermeiros nas CE em puericultura atendem um conjunto de ações sistematizadas e rotineiras, capazes de detectar precocemente alterações na saúde e situações de vulnerabilidades que podem interferir o processo de crescimento e desenvolvimento e, baseado nisso, planejar um cuidado que seja congruente com as necessidades ${ }^{(4)}$.

Em meio a essa contextualização que envolve a CE em puericultura, chama-se a atenção para a crescente demanda de imigrantes haitianos no Brasil, principalmente na Região Sul, sendo Santa Catarina o principal destino, com destaque para quatro municípios catarinenses: Chapecó, Itajaí, Joinville e Blumenau ${ }^{(5)}$, devido à atividade econômica centrada no agronegócio, com grande geração de emprego formal, principalmente nas agroindústrias e construção civil $^{(5-6)}$. Esse fluxo originou-se na presente década, desencadeada após um terremoto que atingiu o Haiti em 2010, seguida de crises humanitárias, altas taxas de desemprego e precárias condições de vida, além da possibilidade de melhoria de vida para os 
familiares que permanecem no Haiti e passam a receber remessas de dinheiro para sobreviver ${ }^{(7)}$.

Diante desse novo cenário, o setor da saúde viu-se diante da necessidade de se modificar e adaptar-se para atender às necessidades de saúde dessa população, dentre as quais, destacam-se as crianças ${ }^{(8)}$.

A maioria das famílias haitianas acessa o Sistema Único de Saúde em busca de atendimento, o que configura uma atividade desafiante aos profissionais, devido à grande diversidade cultural e, muitas vezes, por possuírem dificuldades na intercomunicação. Diante disso, observam-se desafios que merecem atenção, dentre eles, compreender as famílias com base em suas necessidades, particularidades e maneiras diferente de cuidar da saúde das crianças, atentando-se para o fato de que a maioria delas "faz parte de um grupo vulnerável social e economicamente, pois se submetem facilmente a postos de trabalho insalubres, salários baixos e moradias precárias" ${ }^{\text {(6:114) }}$, impactando fortemente na qualidade do cuidado prestado às crianças. Outro desafio vivenciado pelos profissionais é a formação de vínculo, pois as famílias possuem dificuldade de manter a periodicidade de acompanhamento da saúde da criança, muitas vezes, por não conhecerem as Políticas Públicas de Saúde brasileiras e também por entenderem que a busca pelo atendimento nos serviços de saúde deve ocorrer apenas em situações de doença.

Ainda, tendo em vista o aumento da população haitiana no município em estudo, segundo dados coletados no sistema informatizado do município, o WinSaúde ${ }^{(7)}$, residem no município aproximadamente 3.000 haitianos; destes, 629 são crianças. Assim, torna-se necessário, por parte do enfermeiro que realiza as CE de puericultura, conhecer e compreender as necessidades que permeiam a vida dessas famílias, para traçar um plano de ação em conjunto, visando gerar mecanismos para a criação de vínculo e, consequentemente, promover a saúde das crianças.

Tendo em vista o grande aumento da população de haitianos, observada desde $2010^{(7)}$, e a carência de estudos nacionais e internacionais que discutam a atenção à saúde das crianças, especialmente pela ótica do enfermeiro, profissional que está na linha de frente e, muitas vezes, o principal responsável pela puericultura, desenvolveu-se este estudo com o objetivo de identificar quais são as dificuldades vivenciadas pelos enfermeiros na CE em puericultura à criança haitiana na Atenção Primária à Saúde e as possibilidades para superar esses desafios.

Espera-se que os resultados deste estudo possam subsidiar os enfermeiros na compreensão das dinâmicas culturais familiais e, com isso, pensar em estratégias para promover a saúde das crianças nas CE de puericultura.

\section{Método}

Trata-se de estudo exploratório, descritivo, com abordagem qualitativa ${ }^{(9)}$, realizado em um município do Oeste de Santa Catarina com enfermeiros que atuam na assistência direta à criança na Atenção Primária à Saúde.

A seleção dos participantes ocorreu por meio da verificação do registro dos profissionais no Cadastro Nacional de Estabelecimento de Saúde (CNES), sendo identificados inicialmente 53 enfermeiros. Após essa identificação inicial, foram aplicados os critérios de inclusão: atuar diretamente no cuidado à criança no município em estudo, estar cadastrado no CNES, possuir no mínimo seis meses de atuação como enfermeiro. Foram excluídos os que estavam afastados por licença ou férias no momento da coleta dos dados.

Dos 53 enfermeiros cadastrados no CNES, 23 foram excluídos por estarem assumindo, no momento da coleta dos dados, funções gerenciais da Unidade Básica de Saúde (UBS), não realizando ações assistenciais. Quanto aos demais profissionais excluídos do estudo, 2 enfermeiras não possuíam 6 meses de atuação, 1 não aceitou participar da pesquisa e 17 enfermeiros não realizavam atendimento à criança. Desse modo, foram selecionadas 10 enfermeiras que trabalhavam diretamente no cuidado à criança, e foram convidadas a participar mediante contato via telefone e e-mail. 
Quanto ao perfil das enfermeiras participantes do estudo, todas são do sexo feminino, a maioria (8) atuava na APS há mais de quatro anos, duas há menos de dois anos, nove estavam formadas há mais de quatro anos e uma há menos de dois anos. Cinco possuem especialização em Saúde da Família, duas são especialistas em Saúde Pública, uma especialista em terapia intensiva, uma em nefrologia e uma em obstetrícia. Todas atuam como enfermeiras assistenciais em Centros de Saúde da Família (CSF).

A coleta de dados foi realizada no mês de julho de 2018 por meio de entrevistas individuais semiestruturadas, mediante um roteiro pré-estabelecido. Os dados foram submetidos à Análise de Conteúdo ${ }^{(9)}$.

Na pré-análise foi realizada a transcrição dos áudios de cada entrevista, analisando-se as ideias iniciais, de modo a incorporar um esquema para o desenvolvimento das ações sucessivas. A exploração, fase mais longa e aprofundada, consistiu na codificação dos dados. Nessa fase, foram realizadas leituras aprofundadas do material e identificadas as categorias. A fase de tratamento dos resultados, inferência e interpretação teve como característica a validade dos dados brutos, os quais passaram a ter significados ${ }^{(9)}$.

Este estudo integra um macroprojeto de pesquisa intitulado "Estratégias para a implementação da Sistematização da Assistência de Enfermagem no cuidado à mulher e à criança", contemplado pelo Edital da Coordenação de Aperfeiçoamento de Pessoal de Nível Superior/Conselho Federal de Enfermagem (CAPES/COFEN) n. 27/2016. Seguiu as recomendações da Resolução n. 466, de 2012, do Conselho Nacional de Saúde, sendo aprovado pelo Comitê de Ética em Pesquisa (CEP) local, sob Parecer n. 2.630.923, de 2 de maio de 2018.

\section{Resultados}

Os dados gerados pela pesquisa levaram à construção de duas categorias: "Dificuldades na intercomunicação entre enfermeiros e famílias de crianças haitianas" e "Dificuldades culturais relacionadas ao cuidado da criança haitiana". Essas categorias permitiram a identificação de algumas possibilidades para superar os desafios encontrados pelos enfermeiros na realização da CE em puericultura à criança haitiana na Atenção Primária à Saúde.

\section{Dificuldades na intercomunicação entre enfermeiros e famílias de crianças baitianas}

Observa-se que uma das principais dificuldades está relacionada à intercomunicação entre os enfermeiros e as famílias de crianças haitianas, ocasionadas principalmente pela língua estrangeira utilizada pelos haitianos.

[...] a gente não consegue entender muito, a comunicação é difícil. Acho que as haitianas têm dificuldade em entender o que a gente fala também [...]. (E07).

A gente realmente não tem uma boa comunicação pelo fato da lingua. (E08).

[...] acho que é mais a questão da linguagem, onde elas [referindo-se às mães] acabam não entendendo o que tem que ser feito. (E09).

Os enfermeiros identificam as dificuldades encontradas na comunicação como um desafio e, diante dele, sentem a necessidade de adotar medidas diferenciadas de comunicação, como é o caso de desenhar as orientações para facilitar o diálogo com as famílias haitianas e também a necessidade de criar estratégias que possibilitem o acompanhamento dos cuidados propostos pelo enfermeiro na CE em puericultura, como, por exemplo, a busca por essas famílias por meio de visitas domiciliares.

O que a gente cuida, é tentar fazer com que eles compreendam as orientações durante o momento da consulta, pois existe dificuldade na comunicação, tentar fazer um desenbo, tentar fazer com que eles entendam, alguma coisa nesse sentido. (E02).

Acho que as haitianas têm dificuldade em entender o que a gente fala, e tem mais dificuldade de saber a importância, e se a gente não ficar buscando por eles, cuidando, anotando e indo atrás, fazendo visitas domiciliares, eles acabam não seguindo o que foi pedido. (E07).

\section{Dificuldades culturais relacionadas ao cuidado da criança haitiana}

Nesta categoria são discutidas dificuldades relacionadas ao cuidado da criança haitiana influenciadas pelos aspectos culturais. 
Os enfermeiros entendem que existe uma diferença cultural entre haitianos e brasileiros, e que esta pode interferir na dinâmica da CE em puericultura, e consequentemente no cuidado à criança.

\begin{abstract}
Uma cultura totalmente diferente da nossa. Eles não veem a importância da puericultura que para nós é muito importante [...] para eles não é o mesmo sistema. Ainda, na maneira de atender uma mãe nossa [refere-se à mãe brasileira], a abordagem é bem diferenciada. (E07).
\end{abstract}

Realmente os haitianos têm uma cultura diferente, a gente, às vezes, acaba querendo impor algo da nossa cultura, a gente não para e pensa que eles fazem diferente. (E02).

Sob esses aspectos culturais, os profissionais enfermeiros também relatam dificuldades nas demais etapas da CE, como, por exemplo, para encontrar formas que facilitem o entendimento das mães haitianas sobre a importância de alguns cuidados que precisam ter com as crianças no domicílio, para auxiliar no processo de crescimento e desenvolvimento, na promoção da saúde da criança e na prevenção de agravos, além de convencê-las sobre a importância do acompanhamento da criança pelos profissionais de saúde, conforme preconizado pelo Ministério da Saúde (MS).

[...] a gente orienta, mas a gente sabe que muitas vezes eles não vão acatar. (E04).

Precisamos falar sobre todos os cuidados [...] mas nem o básico a gente vê que é feito, as crianças ficam largadas, sujas. (E01).

Simplesmente ela cobre os seios [referindo-se à amamentação], e ela não quer, a reação dela é como uma mãe rejeitando o bebê [...]. Ela não permitiu ensinar a amamentar e ela não está amamentando o bebê. (E07).

[...] a rotina que a família tem, acaba interferindo na questão da consulta, nas orientações de aceitar ou não, porque tem muita gente que acredita que é daquela forma. (E03).

[...] eu acho que muitas vezes a mãe não entende que a puericultura é uma consulta de rotina. (E08).

Os enfermeiros possuem o entendimento de que as questões culturais e econômicas permeiam e influenciam fortemente a adesão à CE em puericultura. Para tentar superar esse desafio reconhecem que precisam ter maiores conhecimentos sobre cada família haitiana, onde moram, seus costumes e quais suas condições econômicas, pois, ao conhecer seu contexto de vida, terão mais elementos para propor os cuidados, como é o caso da alimentação, e também saberão como agir diante de determinadas situações que se apresentam. Além disso, essa aproximação facilita a confiança das famílias e o estabelecimento de vínculos.

Ver qual é a realidade [...] dar algumas orientações, mas tentar ver, direcionar conforme o entendimento, a necessidade dela. (E01).

Eu procuro cuidar, da cultura deles [...] o que eles aceitam o que eles não aceitam, nesse sentido. (E04).

Na área que eu trabalho [referindo-se ao espaço geográfico] eles têm uma dificuldade de entendimento [...] realmente algumas coisas de alimentação, cuidado, a gente tem que ver o que eles têm para poder orientar, aì acaba sempre tendo orientações diferenciadas, conforme a gente conbece a família. (E06).

Olha, normalmente vemos algumas coisas de como é o convivio e tudo mais para facilitar o desenvolvimento da confiança e do vínculo. (E02).

\section{Discussão}

Diante do cenário de imigração dos haitianos no Brasil, que iniciou por volta de 2010, o setor saúde vem passando por grandes modificações e adaptações, por ser um espaço onde o contato com a comunidade é mais próximo, especialmente no atendimento e acompanhamento das necessidades básicas de saúde que ocorre na APS. Com a chegada dos haitianos surgem novos desafios em busca de qualidade na assistência, demandando mecanismos específicos para o atendimento a essa população ${ }^{(8)}$.

Sob essa ótica, para que a população possa usufruir de uma assistência de qualidade, resolutiva e integral, é necessário que usuários e profissionais de saúde tenham conhecimento de seus direitos e deveres. Além disso, as ações desenvolvidas na APS precisam estar ancoradas nos princípios que regem o SUS - universalidade, integralidade e equidade -, garantindo assim, a promoção e a reabilitação da saúde e a prevenção de agravos em todo o ciclo vital humano ${ }^{(7,10)}$. Na perspectiva dos imigrantes, é necessário respeitar a igualdade de acesso aos serviços de saúde, assim como suas características culturais e étnico-raciais, garantindo que os direitos humanos sejam assegurados ${ }^{(7)}$. 
No Brasil, um dos papeis do enfermeiro que atua na Estratégia de Saúde da Família (ESF) é avaliar o estado de saúde da pessoa que busca atendimento, utilizando-se para isso de uma visão holística, voltada para cada usuário, a fim de identificar e compreender agravos, fomentar cuidados de saúde, promover a educação em saúde, planejar e buscar ferramentas para a resolução de problemas ${ }^{(10)}$. Além disso, o enfermeiro necessita compreender a importância de estabelecer vínculos com os usuários e olhar para a demanda atendida, reconhecendo as necessidades de saúde da população assistida com propósitos de intervir de forma eficaz e resolutiva ${ }^{(11)}$.

Tendo em vista essas afirmativas, e pensando especificamente na saúde da criança, o enfermeiro realiza na APS a consulta de puericultura, atividade fundamental para promover o cuidado integral e contínuo, contribuindo para o crescimento e desenvolvimento adequados da criança $^{(12)}$. Contudo, para que a CE em puericultura seja resolutiva, ela precisa ser realizada de forma sistemática e orientada pelas etapas de coleta de dados, diagnóstico de enfermagem, planejamento, implementação e avaliação de enfermagem, conforme preconiza a Resolução COFEN n. 358, de 2009 ${ }^{(13-14)}$.

Entretanto, no estudo aqui apresentado, os enfermeiros que realizam a CE em puericultura na APS, vivenciam algumas dificuldades na sua implementação à criança haitiana. É encontrado, em seus relatos, dificuldades na intercomunicação entre o enfermeiro, a criança e sua família, e que, para driblar a dificuldade com o idioma, precisam buscar outras formas de comunicação, como desenhos. Nessa perspectiva, estudo $^{(15)}$ realizado em um serviço de emergência que atendia haitianos, profissionais de saúde também relataram enfrentar problemas com a comunicação, em virtude dessa dificuldade, e aplicam diferentes estratégias para estabelecer uma comunicação efetiva, como gestos e mímicas. Entretanto, esses profissionais relatam um sentimento de frustração no atendimento prestado aos imigrantes, por considerar que seu atendimento torna-se pouco humanizado, acolhedor e elucidativo ${ }^{(15)}$.
No presente estudo, os enfermeiros não declaram, explicitamente, sentirem-se frustrados, mas afirmam que mesmo utilizando estratégias de comunicação não verbal, como desenhos, acreditam que as mães não seguem os cuidados orientados, também possuem dificuldade de entender que a puericultura é importante e que a avaliação da criança deve ser periódica, e não somente quando a criança já está apresentando algum problema, situação essa, que certamente leva os enfermeiros ao sentimento de frustração.

Acredita-se que a pouca adesão das mães à CE periódica à criança possa ser explicado pelo fato de que, em seu país de origem, o sistema de saúde é pago, situação que leva a população a buscá-lo somente em casos de extrema necessidade; com isso, desenvolvem medidas caseiras para o atendimento dos casos não graves ${ }^{(8)}$.

Estudo realizado em Portugal ${ }^{(16)}$, enfermeiros relataram que também se deparam com dificuldades no atendimento aos imigrantes, enfatizando dificuldades no planejamento de cuidados individualizados, desencadeados pela barreira imposta pela língua falada e, ainda, pelas questões culturais que dificultam a mensagem passada e recebida.

Além disso, estudos ${ }^{(7,15,17)}$ sinalizam que os imigrantes encontram ainda várias dificuldades no acesso aos serviços de saúde, dentre eles o desconhecimento do sistema de saúde vigente no Brasil, medo de utilizar o sistema, principalmente quando ainda estão ilegais, desconhecimento do idioma e diferença cultural que envolve especialmente o cuidado à criança.

Assim como no estudo realizado em Portugal $^{(16)}$, no presente estudo, os enfermeiros também relatam problemas relacionados às questões culturais que permeiam o cuidado infantil. Observam diferenças culturais na forma de cuidar das crianças, principalmente em relação à higiene e à amamentação, dificultando com isso as orientações e os cuidados indicados pelos enfermeiros na CE em puericultura e, consequentemente, causando danos à saúde da criança.

Ao encontro desses achados, outros estudos ${ }^{(15,18-19)}$ também identificaram, no discurso de profissionais da saúde, dificuldades vivenciadas 
pelas diferenças culturais intensificadas por comportamentos inibidos desses imigrantes, bem como pela falta de hábito em procurar os serviços de saúde para prevenção de agravos e promoção de saúde, ou ainda, logo no início dos sinais e sintomas.

Os achados aqui apresentados e discutidos corroboram outros estudos ${ }^{(15-16,18)}$, reforçando as dificuldades no atendimento aos imigrantes pelas questões culturais. Frente a isso, os participantes deste estudo consideram importante, além do acolhimento e da escuta realizada na UBS, conhecer o contexto e a realidade de vida das famílias haitianas, indo ao encontro das considerações do estudo ${ }^{(12)}$, que discute que uma abordagem voltada ao estilo de vida, ao ambiente familiar, à cultura, e à situação econômica da família favorece a promoção de saúde e prevenção de agravos.

É imprescindível ainda, que o enfermeiro, no decorrer das CE em puericultura, valorize os elementos primordiais da família, no seu contexto geral. A valorização das crenças e valores culturais garantem chances de sucesso às orientações realizadas, pois, ao fazer isso, os profissionais de saúde ganham o respeito do usuário e aumentam o índice de resolutividade quanto às necessidades de saúde e doença da criança ${ }^{(20)}$.

Nessa linha de pensamento, especificamente na adesão aos cuidados e orientações propostas na CE em puericultura, os enfermeiros participantes deste estudo entendem que precisam estabelecer orientações diferenciadas, compreender a cultura e os hábitos, para diminuir o máximo possível a possibilidade de não adesão às orientações e ao tratamento. Nesse sentido, estudos $^{(16,20)}$ enfatizam que é importante que a cultura da população atendida seja valorizada, sendo essencial que o enfermeiro tenha conhecimento das práticas que se relacionam ao processo de saúde e doença da criança haitiana, agregando tradições culturais e científicas para que, no momento do acompanhamento da criança, o profissional tenha condições de valorizar os saberes da família, aumentando a confiança. Dessa forma, ao mesmo tempo em que facilita a comunicação para o fornecimento de orientações e a sua apreensão, melhora as chances de que os cuidados e essas orientações sejam seguidos, bem como a CE em puericultura tenha a devida compreensão e valorização ${ }^{(20)}$.

No presente estudo, a necessidade de compreender as questões socioeconômicas e culturais dos imigrantes foi ponto de concordância entre os enfermeiros, que acreditam que, ao se apropriarem dessas questões, conseguirão prestar orientações e cuidados congruentes com a realidade de cada família. Como estratégia para isso, fazem menção à visita domiciliar, pois ela permite conhecer a realidade in loco e, assim, tentar reduzir o distanciamento entre o que é prescrito e aquilo que é possível de ser executado pelas famílias, conforme suas condições econômicas.

A visita domiciliar regular permite ao enfermeiro conhecer o contexto social e cultural da família, seus hábitos e costumes, identificando necessidades. Informações importantes sobre as famílias também podem ser coletadas dos Agentes Comunitários de Saúde (ACS), que são o elo entre a comunidade e os profissionais de saúde, possibilitando o diálogo e a troca de ideias entre os profissionais, melhorando a maneira de intervir no processo de saúde e doença da população alvo ${ }^{(12,21)}$.

A importância de conhecer as condições socioeconômicos foi ponto de discussão em outros estudos $^{(7,15)}$, pois os imigrantes, em sua maioria, encontram-se em situações de vulnerabilidade e fragilidade socioeconômica. Além disso, um dos agravantes dessa situação é que alguns trabalhadores da saúde ainda reproduzem discursos em que os imigrantes são vistos como inferiores, o que compromete o atendimento integral e com equidade ${ }^{(7,15)}$.

Diante do cenário discutido neste estudo, e que reforça outros achados, cabe salientar que a APS é o nível de atenção primordial para fornecer cuidados de saúde à criança haitiana. Contudo, para que isso aconteça, são necessários esforços na realização de ações conjuntas envolvendo os profissionais que atuam nos serviços de saúde e gestores, com vistas a melhorar e qualificar o cuidado à criança, como é direito dos imigrantes ${ }^{(8,22)}$. 
Assim, para superar os desafios encontrados no atendimento às crianças, além das possibilidades apresentadas pelos enfermeiros que participaram deste estudo, autores ${ }^{(15,22-23)}$ discutem outras alternativas que podem facilitar a assistência integral e resolutiva. Indicam a possibilidade de ampliar o olhar para a criança por meio de ações comunitárias na APS, objetivando concretizar atividades interdisciplinares que visam garantir ações de promoção e educação em saúde, como, por exemplo, o acompanhamento dessas crianças nos espaços de educação infantil e creches.

Outra possibilidade para melhorar a intercomunicação e o vínculo dos imigrantes com os profissionais de saúde, seria a contratação de imigrantes para atuar como ACS, como demonstra estudo ${ }^{(24)}$, que aborda essa prática como facilitadora do acesso dos imigrantes às ações realizadas pelas ESF, bem como melhor compreensão da inserção dessa população no território e suas características socioculturais.

Além disso, a criação de tecnologias educativas, do tipo folder, com informações sobre o sistema de saúde e orientações sobre cuidados fundamentais no idioma dos imigrantes apresenta-se como estratégia que visa facilitar a intercomunicação ${ }^{(24)}$.

Ainda, outra estratégia que se apresenta como uma possibilidade de aproximação e entendimento às necessidades da população de imigrantes é a formação permanente dos enfermeiros e demais profissionais das ESF voltada para a comunicação, compreensão dos aspectos étnico-raciais e as necessidades dessa população, sobretudo no cuidado à criança ${ }^{(15)}$.

As estratégias apresentadas nesta pesquisa e em outros estudos podem contribuir para a atuação do enfermeiro, auxiliando-o nas dificuldades encontradas, especialmente na realização da CE em puericultura na APS, com vistas a garantir o acesso aos serviços de saúde pelos imigrantes, por meio de acolhimento humanizado, em acordo com as necessidades da criança e sua família, respeitando suas características étnico-culturais e promovendo o cuidado integral à saúde da criança haitiana.
Como limitação desta pesquisa, destaca-se a escassez de estudos sobre a temática, especialmente envolvendo a CE da criança imigrante, sobretudo da criança haitiana. A grande maioria dos estudos possui como foco a avaliação das condições de empregabilidade dos imigrantes haitianos e sua disposição geográfica no Brasil. Diante disso, considera-se que as discussões realizadas neste estudo são extremamente relevantes e indicam a necessidade de se implementar ações de educação permanente, para que os profissionais de saúde possam qualificar-se para esse atendimento, com vistas a melhorar a adesão à CE de puericultura e, consequentemente, ter subsídios para o desenvolvimento de ações de promoção da saúde aos imigrantes.

\section{Conclusão}

O estudo revela as dificuldades vivenciadas pelos enfermeiros para a realização da CE à criança haitiana. Dentre as principais dificuldades, destacam-se a intercomunicação, a adesão das famílias à CE em puericultura e o seguimento às orientações realizadas pelos enfermeiros. Dificuldades influenciadas pela língua e pela cultura dos haitianos, que entendem a necessidade de buscar pelo atendimento de um profissional de saúde somente em situações de doença.

Outro ponto de destaque, é que a cultura haitiana é pouco conhecida pelos profissionais de enfermagem, o que dificulta suas ações e a tomada de decisão para um cuidado congruente com as necessidades da criança e a realidade das famílias. Essa situação remete à necessidade de instituir atividades de educação permanente em saúde, visando subsidiar os profissionais de saúde para qualificação do atendimento a essa população, cada vez maior no Brasil.

Para superar as dificuldades vivenciadas, os enfermeiros apresentam algumas estratégias como a comunicação não verbal, a exemplo de desenhos, visita domiciliar de rotina para acompanhar os cuidados e o cumprimento das orientações prescritas e também para conhecer o contexto de vida - moradia, costumes, hábitos -, 
e, com isso, aproximar-se das famílias, ganhar confiança e estabelecer vínculos.

\section{Colaborações:}

1 - concepção, projeto, análise e interpretação dos dados: Elisangela Argenta Zanatta, Cheila Karei Siega, Ingrid Pujol Hanzen e Luiza Alcântara de Carvalho;

2 - redação do artigo e revisão crítica relevante do conteúdo intelectual: Elisangela Argenta Zanatta e Cheila Karei Siega;

3 - aprovação final da versão a ser publicada: Elisangela Argenta Zanatta, Cheila Karei Siega, Ingrid Pujol Hanzen e Luiza Alcântara de Carvalho.

\section{Referências}

1. Abud SM, Gaíva MAM. Registro dos dados de crescimento e desenvolvimento na caderneta de saúde da criança. Rev Gaúcha Enferm. 2015;36(2):97-105. DOI: http://dx.doi.org/10.1590/ $1983-1447.2015 .02 .48427$

2. Brasil. Ministério da Saúde. Secretaria de Atenção Básica à Saúde. Departamento de Atenção Básica. Saúde da Criança: crescimento e desenvolvimento [Internet]. Brasília (DF); 2012. (Cadernos de Atenção Básica, no 33) [cited 2019 Jun 19]. Available from: http://189.28.128.100/dab/docs/ publicacoes/cadernos_ab/caderno_33.pdf

3. Carvalho EB, Sarinho SW. A Consulta de Enfermagem no acompanhamento do crescimento e desenvolvimento de crianças na Estratégia Saúde da Família. Rev enferm UFPE on line. 2016;10(Suppl 6):4804-12. DOI: 10.5205/ reuol.8200-71830-3-SM.1006sup201612

4. Duarte ED, Silva KL, Tavares TS, Nishimot CLJ, Walty CMRF, Sena RR. Desafios do trabalho da enfermagem no cuidado às crianças com condições crônicas na atenção primária. Esc Anna Nery. 2015;19(4):648-55. DOI: 10.5935/ $1414-8145.20150087$

5. Cavalcanti L, Brasil El, Dutra D. A movimentação dos imigrantes no mercado de trabalho formal: admissões e demissões In: Cavalcanti L, Oliveira T, Araujo D, organizadores. Relatório Anual 2016. A inserção dos imigrantes no mercado de trabalho brasileiro [Internet]. Brasília (DF): Observatório das Migrações Internacionais; 2016 [cited 2020 Apr 10].
Available from: https://portaldeimigracao.mj.gov. br/images/dados_anuais/RelatorioCompleto_ v8_0512_pagespelhada_comcapa.pdf

6. Cavalcanti L, Tonhati T. Características sociodemográficas e laborais da imigração haitiana no Brasil. Périplos: Rev estudos migrações [Internet]. 2018 [cited 2019 Oct 15];1(1):68-71. Available from: http://periodicos.unb.br/index.php/obmigra_ periplos/article/view/5882

7. Risson AP, Matsue RY, Lima ACC. Atenção em Saúde aos Imigrantes Haitianos em Chapecó e suas Dimensões Étnico-Raciais. Soc Quest [Internet]. 2018 [cited 2019 Oct 22];31(41):111-30. Available from:http://osocialemquestao.ser.puc-rio.br/media/ OSQ_41_art_5_Risson_Matsue_Lima.pdf

8. Risson A. Cartografia da atenção à saúde de imigrantes haitianos residentes em Chapecó, SC [dissertação]. Chapecó (SC): Universidade Comunitária da Região do Chapecó; 2015 [cited 2019 May 22]. Available from: https://laemiceppac. files.wordpress.com/2015/07/projeto-mestradoana-risson.pdf

9. Bardin L. Análise de conteúdo. São Paulo: Edições 70; 2016.

10. Furtado MCC, Mello DF, Pina JC, Vicente JB, Lima PR, Rezende VD. Ações e articulações do enfermeiro no cuidado da criança na atenção básica. Texto contexto - enferm. 2018;27(1):e0930016. DOI: http://dx.doi.org/10. 1590/0104-07072018000930016

11. Santos LNM, Pedrosa JIS, Rodrigues IDCV, Freire MSS, Silva GRF, Luz MHBA. Relações interpessoais nas estratégias saúde da família: reflexo na qualidade dos cuidados de enfermagem. Rev enferm UFPE on line. 2014;8(1):155-9. DOI: 10.5205/reuol.4843-39594-1-SM.0801201421

12. Góes FGB, Silva MA, Paula GK, Oliveira LPM, Mello NC, Silveira SSD. Contribuições do enfermeiro para boas práticas na puericultura: revisão integrativa da literatura. Rev bras enferm. 2018;71(Suppl 6):2808-17. DOI: http://dx.doi. org/10.1590/0034-7167-2018-0416

13. Conselho Federal de Enfermagem. Resolução no 358/2009, de 15 de outubro de 2009. Dispõe sobre a Sistematização da Assistência de Enfermagem e a implementação do Processo de Enfermagem em ambientes públicos ou privados, em que ocorra o cuidado profissional de Enfermagem [Internet]. Brasília (DF); 2009 [cited 2019 Dec 10]. Available from: http://www.cofen. gov.br/resoluo-cofen-3582009_4384.html 
14. Almeida ER, Moutinho CB, Carvalho SAS, Araújo RN. Relato sobre a construção de um protocolo de enfermagem em puericultura na atenção primária. Rev enferm UFPE on line. 2016;10(2):683-91. DOI: 10.5205/reuol. 8557-74661-1-SM1002201640

15. Barreto MDS, Nascimento DGD, Magini LYZ, Oliveira ILD, Vieira VCDL, Marcon SS. Discurso de enfermeiros e médicos sobre a utilização do serviço de emergência por imigrantes. Esc Anna Nery. 2019;23(3):e20190003. DOI: http://dx.doi. org/10.1590/2177-9465-ean-2019-0003

16. Reis ASC, Costa MAM. Cuidar de Imigrantes: das interações em contexto à construção de competências culturais nos enfermeiros. Rev Enf Ref. 2014;serIV(2):61-69. DOI: http://dx.doi. org/10.12707/RIII13118

17. Santos FV. A inclusão dos migrantes internacionais nas políticas do sistema de saúde brasileiro: o caso dos haitianos no Amazonas. Hist ciênc saúdeManguinhos. 2016;23(2):477-94. DOI: http:// dx.doi.org/10.1590/S0104-59702016000200008

18. Kanengoni B, Andajani-Sutjahjo S, Holroyd E. Setting the stage: reviewing current knowledge on the health of New Zealand immigrants - an integrative review. Peer J. 2018;6:e5184. DOI: https://doi.org/10.7717/peerj.5184

19. Mahmoud I, Eley R, Hou XY. Subjective reasons why immigrant patients attend the emergency department. BMC Emerg Med. 2015;15(4):1-6. DOI: https://doi.org/10.1186/s12873-015-0031-8
20. Moreira MDS, Gaíva MAM. Abordagem do contexto de vida da criança na consulta de enfermagem. Rev Pesqui. 2017;9(2):432-40. DOI: http://dx.doi. org/10.9789/2175-5361.rpcfo.v9.5433

21. Kerbian LVA, Acioli S. A visita domiciliar de enfermeiros e agentes comunitários de saúde da Estratégia Saúde da Família. Rev eletrônica enferm. 2014;16(1):161-9. DOI: http://dx.doi.org/10.5216/ ree.v16i1.20260

22. O'Donnell CA, Burns N, Mair FS, Dowrick C, Clissmann C, van den Muijsenbergh $\mathrm{M}$, et al. Reducing the health care burden for marginalised migrants: the potential role for primary care in Europe. Health Policy. 2016;120(5):495508. Available from: https://doi.org/10.1016/j. healthpol.2016.03.012

23. Reichert APS, Leônico ABA, Toso BRG, Santos NCCB, Vaz EMC, Collet N. Orientação familiar e comunitária na Atenção Primária à Saúde da criança. Ciênc saúde colet. 2016;21(1):119-27. DOI: http://dx.doi.org/10.1590/ 1413-81232015211.05682014

24. Aguiar ME, Mota A. O Programa Saúde da Família no bairro do Bom Retiro, SP, Brasil: a comunicação entre bolivianos e trabalhadores de saúde. Interface. 2014;18(50):493-506. DOI: http:// dx.doi.org/10.1590/1807-57622013.0040

Recebido: 16 de março de 2020 Aprovado: 11 de maio de 2020 Publicado: 15 de junho de 2020

A Revista Baiana de Enfermagem utiliza a Licença Creative Commons - Atribuição-NãoComercial 4.0 Internacional. https://creativecommons.org/licenses/by-nc/4.0/

Este artigo é de acesso aberto distribuído sob os termos da Licença Creative Commons (CC BY-NC). Esta licença permite que outros remixem, adaptem e criem a partir do seu trabalho para fins não comerciais. Embora os novos trabalhos tenham de lhe atribuir o devido crédito e não possam ser usados para fins comerciais, os usuários não têm de licenciar esses trabalhos derivados sob os mesmos termos. 